\title{
A Reduction in Time with Electronic Monitoring In Stroke trial
}

Gaia T. Koster ${ }^{1}$, Adrien E. Groot ${ }^{2}$, T. Truc My Nguyen ${ }^{1}$, Jonathan M. Coutinho², Jan Bosch ${ }^{3}$, Heleen M. den Hertog ${ }^{4}$, Ale Algra ${ }^{5,6}$, Marieke Wermer ${ }^{1}$, Yvo B. Roos², Nyika Kruyt ${ }^{1}$, for the ARTEMIS investigator group.

${ }^{1}$ From the Department of Neurology, Leiden University Medical Center Leiden. ${ }^{2}$ From the Department of Neurology, Academic Medical Center Amsterdam. ${ }^{3}$ RAV Hollands Midden, Leiden. ${ }^{4}$ Department of Neurology, Medical Spectrum Twente, Enschede. ${ }^{5}$ Department of Clinical Epidemiology, Leiden University Medical Center, Leiden. ${ }^{6} J u l i u s$ Centre, University Medical Center, Utrecht.

\section{BACKGROUND}

For the clinical benefit of intravenous thrombolysis (IVT) and intraarterial thrombectomy (IAT), time is the most crucial factor.

The delay from alarming the Emergency Medical Services (EMS) dispatch office until initiation of reperfusion therapy depends on logistics and collaboration between various caregivers in this trajectory (i.e. total system delay [TSD], see Figure 1).

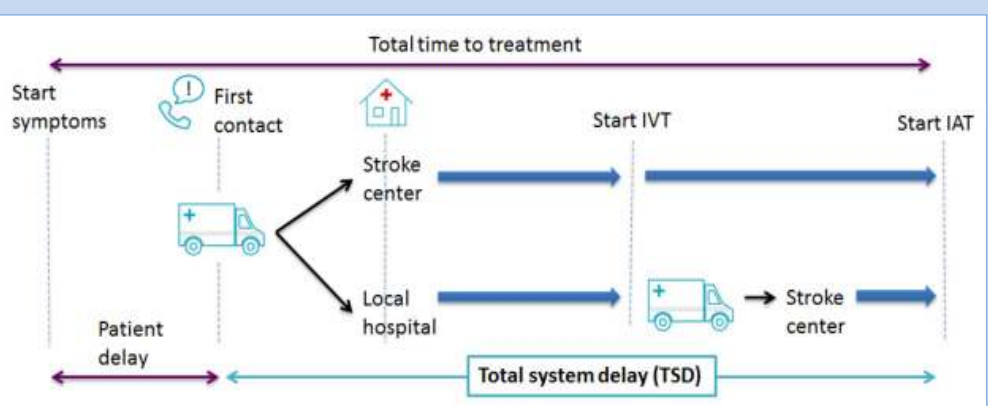

Figure 1. Schematic overview of the acute care trajectory for patients

\section{METHODS}

Design: Multicenter, multicenter, randomized open end-point trial.

Inclusion criteria: all consecutive patients over 18 years with suspected stroke and eligibility for IVT and/or IAT, identified by the EMS dispatch office or by paramedic on-site.

츨 Run-in phase: implementation of hardware and time registration will first be tested during the run-in phase.

슬 Randomization phase: real-time audio-visual feedback on actual TSD will be provided to caregivers. Randomization will be per patient.

슨 Sample size: by including 75 IAT and 225 IVT patients in each arm, we will be able to demonstrate a 20-minute reduction on TSD to IAT and a 10-minute reduction on TSD to IVT ( $\alpha=0.05$ and power $=0.8$ ). with a suspected stroke

\section{AUTOMATIC TRACKING SYSTEM}

Patients will receive a wristband with an iBeacon which emits a Bluetooth signal. Handhelds and tablets along the route will pick-up this signal and register the time. The following times will be recorded:

- First contact EMS

- Arrival of ambulance at patient

- Departure of ambulance to hospital

- Door hospital

- Shock room/CT-room hospital

- Needle (IVT)

- Arrival at angio suite

- Groin puncture (IAT)

- Moment of reperfusion or last angio run (IAT).

\section{INTERVENTION}

Real-time audio-visual feedback to caregivers on actual TSD

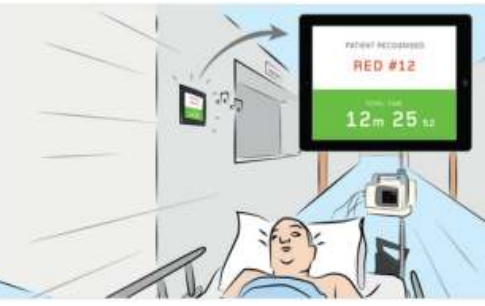

\section{OUTCOME MEASURES}

Primary outcome: Total System Delay to IVT and IAT.

Secondary outcomes: percentage of patients treated with IVT and/or IAT, functional outcome after 90 days (measured with modified Rankin Scale score), complications (e.g. symptomatic intracranial hemorrhage) and cost-effectiveness.

\section{RESULTS}

Total number of current inclusions: 13 patients (12 IVT and 5 IAT)

Leiden and Amsterdam are currently recruiting patients.

Twente will start in June 2018.

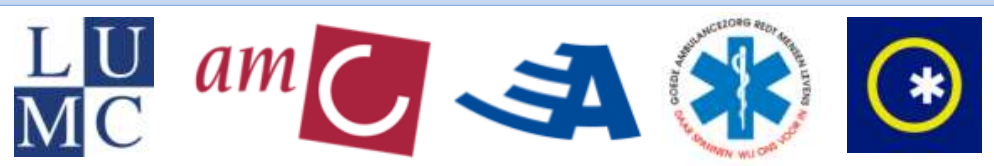

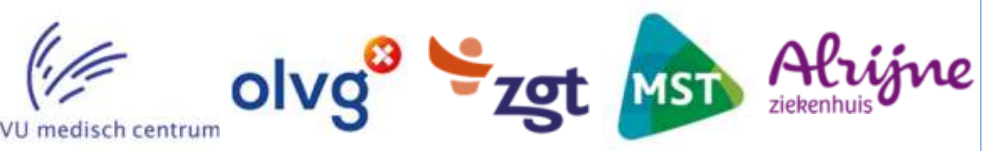

ARTEMIS investigator group: A. Algra, J. Bosch, J.M. Coutinho, A.E. Groot, H.M. den Hertog, G.T. Koster, N.D. Kruyt, V.I.H. Kwa, T.T.M. Nguyen, Y.B.W.E.M. Roos, S.M. van Schaik, E.L.L.M. de Schryver, M.C. Visser, M.A.A. van Walderveen, M.J.H. Wermer.

9w. ww.artemistrial.org artemis@amc.uva.nl

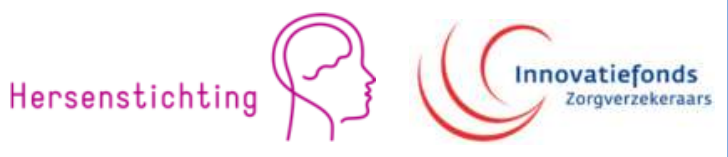

